\section{Up or Down?}

THE assertion of the report of the Royal Commission on Population in 1949 that there would be "a substantial decline in the number of births over the next 15 ycars" has often been contrasted with the rise in the number of births in Great Britain during the 1950s.

How far has the science of population projection progressed in the 20 years since such estimates were first made? This question must be in the mind of anyone reading the article on recent changes in the birth rate of England and Wales by Eric Thompson, chief statistician of the Research and Intelligence Unit of the Greater London Council, in the current issue of Flamily Planning (18, No. 4 ; 1970). Thompson discusses the decline in the number of births in the past 5 years and the assumptions made about this decline by the Government Actuary in preparing the official population projections for England and Wales to the year 2001. In preparing the projections based on mid-1968, the Registrar-General commented that the post-1964 downward trend secmed to be slowing and was regarded as a temporary aberration in a secular increase. Thompson maintains that this is not so and that recently births have returned to the post-1964 downward trend.

The decline in the number of births since 1964 about 8 per cent in the 5 years to 1969 -has been unexpected. Most of the variables which influence the number of births have been moving in ways which would lead to an expectation of increasing births. 'The number of women aged 20 to 29 increased by 11 per cent between 1964 and 1968 and will increase to 30 per cent over the 1964 figure by the mid-1980s. If the birth rate had remained constant at its 1964 level, this increase in the number of women in the most fertile age group would have led to a total of births in 1969 about 15 per cent greater than that which actually occurred. Age at marriage is decreasing, and because more than 90 per cent of births occur within marriage, other things being equal, this increases the percentage of women in the fertile age groups "at risk" of pregnancy. Changes in the proportion of women married at any particular age will have a short term effect on the birth rate because the range of potential changes is obviously circumscribed at both ends of the age range, more especially at the younger ages, but changes in the mcan completed family size of marriages contracted at different times can have a more profound effect on the total of births.

The downward trend in the number of births forced a revaluation of the assumptions about mean completcd family size for marriages recently contracted and still to be contracted. In particular, the positive differential in mean completed family size for marriages contracted at an early age (under 20 ) has been reduced because fertility at early ages has not continued to increase as it did during the past decade. There seems to be a convergence with the decreasing fertility of older ages. Thompson wonders whether the next set of projections should incorporate a more substantial narrowing of the early marriage-late marriage fertility differential, and questions whether it is correct to assume any increase in mean completed family size for current marriages.

It is hard to escape from the fact that the 5 year decline in the number of births is really too short a period on which to base any conclusive arguments. The reduced number of children born during 1964-69 is, however, a fact and must have an effect on the future age structure, and, from about 1984 onwards, on the level of future births. The reduction in the strength of the relationship between early marriage and high fertility seems to be the key to the enigma of the current pattern of number of births. Detailed studies alone will reveal the motivation behind this trend and it is important for assessing the future number of births to discover whether this relationship will soon stabilize somewhere near current levels, or whether the decline will continue until the marriage age differential in completed family size becomes much less marked than at present.

\section{SEYFERT GALAXIES}

\section{A Cornucopia of Matter}

\section{by our Astronomy Correspondent}

SEYFER'I galaxies with their extraordinarily bright and unusual nuclei could easily be intermediaries between quasars and normal galaxies. Indeed, part of the evidence is that even superficially normal galaxies such as the Milky Way have a hint of abnormal. ity about them. Yet it is far from settled in which direction the evolution is going. Obviously the morf that can be found out about Seyferts the better, and in the current issue of Astrophysical Journal Kurt Anderson and Robert Kraft of Lick Observatory present evidence that matter is being ejected from the nucleus of the Seyfert galaxy NGC 4151 (158, 859; 1969).

They have shown that the nucleus of NGC 4151 seems to be losing mass at a rate which raises questions about where it is all coming from. Making several 